\title{
Original Research \\ Impact of the COVID-19 pandemic on the policies and practices of delivery facilities in Japan
}

\author{
Yasuhiko Ebina ${ }^{1, *}$, Mizuki Takeuchi ${ }^{2}$, Maya Nakamura ${ }^{1}$ \\ ${ }^{1}$ Division of Comprehensive Development Nursing, Faculty of Health Sciences, Hokkaido University, 060-0812 Sapporo, Hokkaido, Japan \\ ${ }^{2}$ Graduate School of Health Sciences, Hokkaido University, 060-0812 Sapporo, Hokkaido, Japan \\ *Correspondence: ebiyas@hs.hokudai.ac.jp (Yasuhiko Ebina) \\ Academic Editor: Luca Roncati \\ Submitted: 12 October 2021 Revised: 4 December 2021 Accepted: 13 December 2021 Published: 10 January 2022
}

\begin{abstract}
Background: To clarify the impact of the COVID-19 pandemic on the policies and practices of delivery facilities in Japan from April 2020 to March 2021. Methods: We conducted an online survey of midwives working in obstetric wards nationwide between May 15, 2021, and July 31, 2021. We analyzed the 376 valid responses. Results: With the COVID-19 pandemic, most facilities have barred or partially restricted families and other visitors from being present (patient companions) in outpatient clinics, prenatal classes, during delivery, inpatient care, and inpatient visits. These changes were implemented nationwide, regardless of regional differences in the pandemic state of COVID-19. Meanwhile, by facility classification, prenatal classes, companion at birth, and inpatient visits were more likely to be canceled at perinatal centers than at clinics. The acceptance of "satogaeri deliveries" (a custom in Japan where pregnant women return to their natal homes for labor and childbirth) was partially restricted to about $40 \%$ of facilities, although this rate was lower in the four Kanto prefectures (Tokyo, Kanagawa, Saitama, and Chiba). Conclusions: With the COVID-19 pandemic, the differences in Japan's policies and practices of delivery facilities were attributed more to the role played by facilities in the regional perinatal system than to regional pandemic status.
\end{abstract}

Keywords: COVID-19 pandemic; Delivery facility; Health care surveys; Maternal-child health centers; Maternity hospital

\section{Introduction}

In Japan, cases of novel coronavirus infection (COVID-19) have been reported since January 2020, and the number has been increasing steadily. Therefore, on April 7, a COVID-19 state of emergency was declared for seven prefectures, including Tokyo, and people were requested to refrain from going out for non-essential and/or non-urgent reasons and at night. After that, on April 16, the state of emergency measures expanded to include all prefectures. Since this policy limited citizens from moving across prefectures, pregnant women were not able to return to their hometowns across prefecture borders to have a "satogaeri delivery". "Satogaeri delivery" is Japan's unique birthing custom. Returning to the parents' home when the expected delivery date is approaching is called "satogaeri". In general, a woman will stay at her parents' home from about one month before delivery until about one to three months after delivery to receive assistance with childcare and other matters. During this time, the husband goes to the woman's parents' house or spends weekends with them. On May 4 , the state of emergency measures for all prefectures was further extended until May 31. Subsequently, the number of new COVID-19 cases began to decrease, and from May 14 , only eight prefectures continued the state of emergency measures. The state of emergency was then lifted nationally on May 25. However, on May 26, the Japan Society of Obstetrics and Gynecology and the Japan Association of Obstetricians and Gynecologists announced that "longdistance travel has a high risk of infection; as such, refrain from 'satogaeri delivery' as much as possible even after the state of emergency is lifted".

Japan's perinatal system differs somewhat from that in other developed countries. More than 2000 delivery facilities deal with 860,000 deliveries annually (2019). Over half the facilities are private clinics with one or two obstetricians. A general hospital manages high-risk pregnancies and deliveries, and a perinatal center handles premature births. In each region of Japan, clinics, general hospitals, and perinatal centers have different functions and collaborate with each other. COVID-19 infection prevention measures were implemented at various delivery facilities. Patient companions during delivery and inpatient visits were restricted, and the situations surrounding pregnancy and childbirth changed drastically. To date, there have been reports of COVID-19 infection control and medical fact-finding surveys at delivery facilities in Japan [1-3]. However, no research is available investigating the impact of COVID-19 on the unique perinatal system in Japan described above.

It is important to clarify the changes in the healthcare system related to pregnancy and delivery following the COVID-19 pandemic in order to design future strategies. In May 2021, we conducted a nationwide questionnaire of midwives at delivery facilities regarding preventive inter- 
vention during pregnancy for postpartum depression. Since it was necessary to consider the impact of the COVID-19 pandemic in implementing this intervention, we also asked how the COVID-19 pandemic altered the policies and practices of each facility. Therefore, in this paper, among the results obtained in this survey, we also clarify the actual state of the effects of the COVID-19 pandemic on the policies and practices of delivery facilities.

\section{Methods}

An online survey was used in this study. As a "national survey on preventive interventions for postpartum depression", a survey request form and a form with the URL/QR code for Google Forms were sent to the head nurse of the obstetrics/gynecology departments at delivery facilities throughout Japan on May 10, 2021. The respondents were midwives with $>6$ years of clinical experience working in the obstetrics/gynecology ward, and one anonymous response from one facility was requested. The survey questions consisted of the attributes of the subjects and obstetric facilities, the implementation status of preventive interventions for postpartum depression, and the impact of the COVID-19 pandemic on the obstetric medical system and intervention implementation. Regarding the affiliation facility, the location was selected from 47 prefectures, and the classification of medical institutions was selected (clinics, hospitals (including departments other than obstetrics/gynecology), hospitals (obstetrics/obstetrics and gynecology only)), designated categories of facilities (comprehensive perinatal maternal and child medical center, regional perinatal maternal and child medical center, not applicable). The survey was conducted between May 15, 2021, and July 31, 2021.

Questions regarding maternal care during the COVID19 pandemic were phrased as follows:

"Please select all that apply regarding changes in the obstetric medical system due to the pandemic and spread of COVID-19. Please tell us about the biggest changes between April 2020 and March 2021. (Example) If prenatal classes were canceled once before but are now taking place under certain restrictions $\rightarrow$ Select [Canceled]".

The following nine policies and practices of delivery facilities were surveyed: (1) acceptance of "satogaeri deliveries", (2) outpatient visits for pregnant women, (3) companion for outpatient visits, (4) prenatal classes, (5) companion for delivery, (6) companion during inpatient care (family hospitalization), (7) inpatient visits, (8) postpartum outpatient visits, and (9) postnatal classes. Regarding the influence on each, the participants were asked to select one from (1) canceled, (2) partial restriction, (3) no change, and (4) not applicable.

For the analysis, facilities were classified into one of three groups - perinatal centers, hospitals other than perinatal centers, and clinics - by combining the type of medical facility (hospital or clinic) and perinatal center designation.
This classification considers the role of facilities in the perinatal system of each region. Perinatal centers include comprehensive perinatal maternal and child medical centers and regional perinatal maternal and child medical centers. Furthermore, to see the difference in the effects of the COVID19 pandemic in each prefecture, the number of days under the state of emergency between April 2020 and March 2021 was used to categorize prefectures into one of three groups: high, middle, and low pandemic regions (Supplementary Fig. 1). Then, the above nine items were totaled for all facilities, compared according to the facility classification, and compared according to three regions. Statistical analysis was performed using SPSS Statistics version 26 (IBM Corp., Armonk, NY, USA) using the $\chi^{2}$ test, and statistical significance was set at $p<0.05$.

\section{Results}

A request form was sent to 2061 facilities, and 390 responses to Google Forms were received within the survey period (response rate: 18.9\%). After excluding duplicate responses, 376 responses were regarded as valid responses, and the following analysis was performed. Responses were received from facilities in all 47 prefectures. Supplementary Table 1 shows the breakdown of medical institution classification, facility designation, and area classification of regions and prefectures. The median age of the respondents was 47 years (range: 27-73 years), and the number of years of clinical experience was 22 years (6-40 years).

Fig. 1 shows the changes in the policies and practices of delivery facilities due to the COVID-19 pandemic. A decrease in the acceptance of "satogaeri deliveries" was seen in $42.6 \%$ of the facilities, including cancelation $(5.6 \%)$ and partial restrictions (37.0\%). Outpatient visits for pregnant women refer to health checkups for pregnant women. A total of $69.4 \%$ remained unchanged. Partial restrictions were made in $23.9 \%$ of cases, while cancelation was seen in $1.3 \%$. Companions during outpatient visits refers to a spouse or family member accompanying a patient during an outpatient visit. A total of $87.2 \%$ of facilities were affected, including cancelation (52.1\%) and partial restrictions $(35.1 \%)$. Prenatal classes refer to maternity and parenting classes. Of these, $69.1 \%$ were canceled, and $23.1 \%$ had partial restrictions, with a total of $92.2 \%$ affected. A total of $66.8 \%$ of facilities canceled companions during delivery, and $28.5 \%$ partially restricted them. A total of $80.6 \%$ of facilities canceled companions during inpatient care (family hospitalization). A total of $78.7 \%$ facilities canceled inpatient visitation. A total of $67.0 \%$ postpartum outpatient visits were unchanged, $23.1 \%$ had partial restrictions, and $3.7 \%$ were canceled. Postpartum classes refer to postpartum support in the delivery facilities. Many facilities were not applicable; excluding these non-applicable facilities, $49.0 \%$ were canceled, and $27.6 \%$ had partial restrictions. 


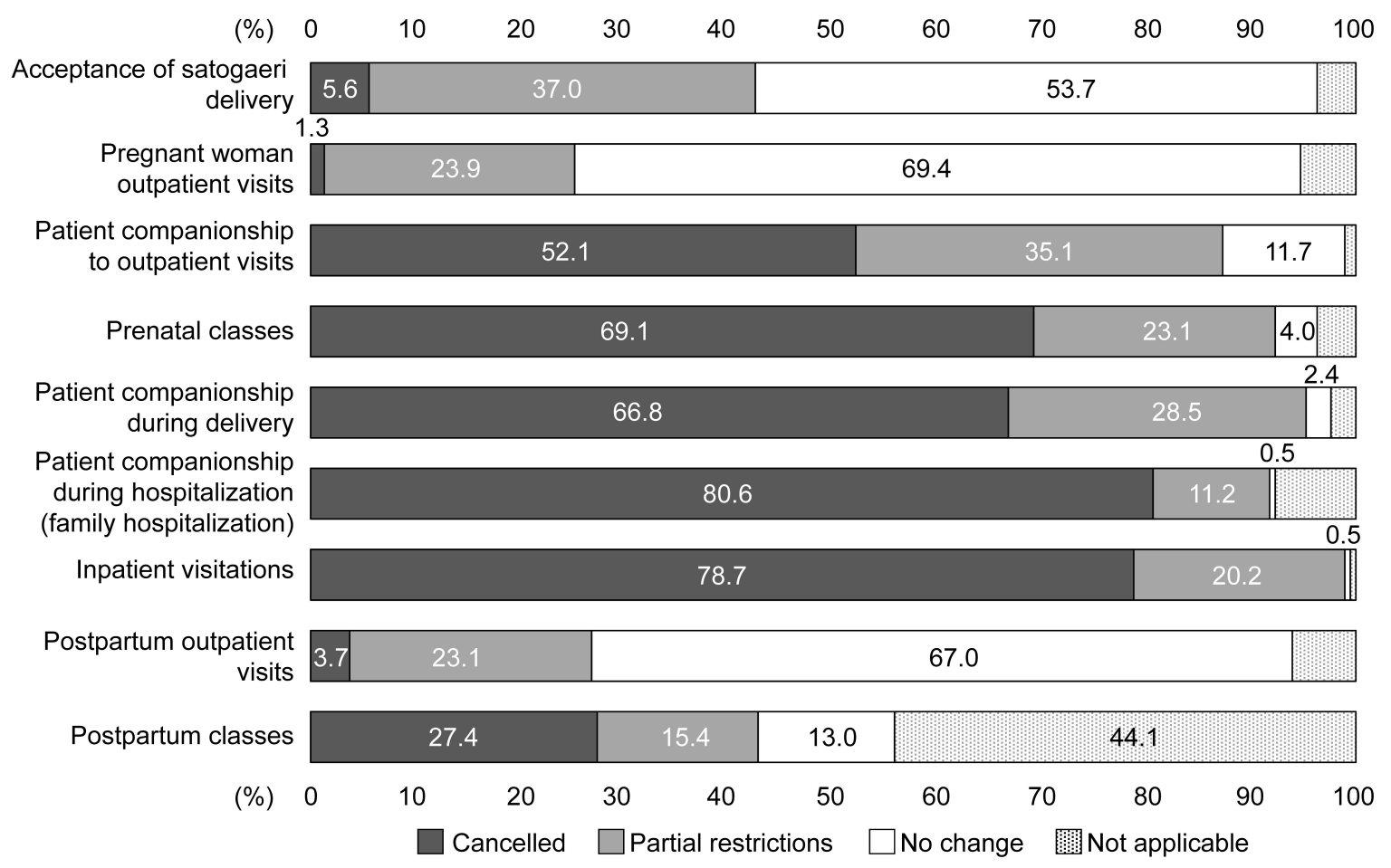

Fig. 1. Changes in the policies and practices of delivery facilities in Japan due to COVID-19 pandemic (376 facilities).

Fig. 2 shows the changes in the policies and practices of delivery facilities due to COVID-19 pandemic by facility classification. No differences in acceptance of "satogaeri deliveries" were found according to facility classification. At hospitals other than centers, 7.0\% canceled "satogaeri deliveries", 36.4\% had partial restrictions, and 54.3\% had no changes. At clinics, $82.6 \%$ canceled companions during outpatient visits, and $15.9 \%$ had partial restrictions. Clinics tended to cancel companions more often than the other two categories of facilities. Perinatal centers $(80.7 \%)$ and hospitals other than the centers $(73.6 \%)$ tended to have more cancelations. Companions during delivery were canceled in $84.8 \%$ of perinatal centers and in $69.8 \%$ of hospitals other than centers. On the other hand, $50.0 \%$ of clinics canceled companions during delivery, and $46.4 \%$ had partial restrictions. Fewer clinics tended to cancel inpatient visitation $(65.9 \%)$ than the other two categories of facilities. There were no differences between facility categories for outpatient visits of pregnant women, companions during hospitalization, postpartum outpatient visits, and postpartum classes.

When the nine items of the policies and practices were compared by dividing them into the three regions shown in Supplementary Fig. 1, a difference was found only in the acceptance of "satogaeri deliveries". Approximately 4.5\% of facilities canceled "satogaeri deliveries", which was not significant, but there was a difference in the percentage of partial restrictions. Partial restrictions in middle pandemic region were $41.2 \%$, and partial restrictions in low pandemic region were $44.0 \%$, both of which tended to be higher than that in the high pandemic region. In contrast, the ratio of facilities that did not change was $78.2 \%$ in the high pandemic region, which tended to be higher than that in middle and low pandemic regions (Fig. 3).

Regarding the facilities in the three COVID-19 pandemic status that had a difference in the acceptance of "satogaeri deliveries", the facility categories were combined and examined. At the clinics, no differences were observed according to pandemic status. At the perinatal centers, the proportion of facilities with partial restrictions tended to increase in the order of high $\rightarrow$ middle $\rightarrow$ low pandemic regions. In many general hospitals/clinics, there was no change in restrictions in the high pandemic region. In both middle and low pandemic region, the proportions of facilities with partial restrictions and no change were similar (Fig. 4).

\section{Discussion}

This survey elucidated the impact of the COVID-19 pandemic on the policies and practices of delivery facilities during one year from April 2020, when the first COVID-19 state of emergency was declared in Japan.

The acceptance of "satogaeri deliveries" was almost the same in all regions except in the four prefectures in the Greater Tokyo Area. Around $4-6 \%$ of the facilities canceled them, and the rest had almost the same rate of partial restrictions and no change in acceptance. On the other hand, there was no difference in the acceptance status by facility classification, such as perinatal centers. According to a survey conducted by the Ministry of Health, 
A

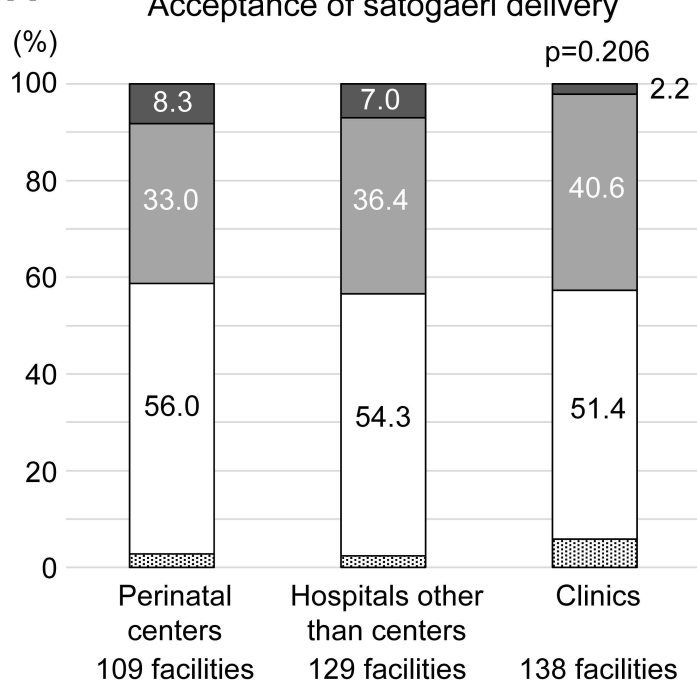

B Patient companionship in outpatient visits

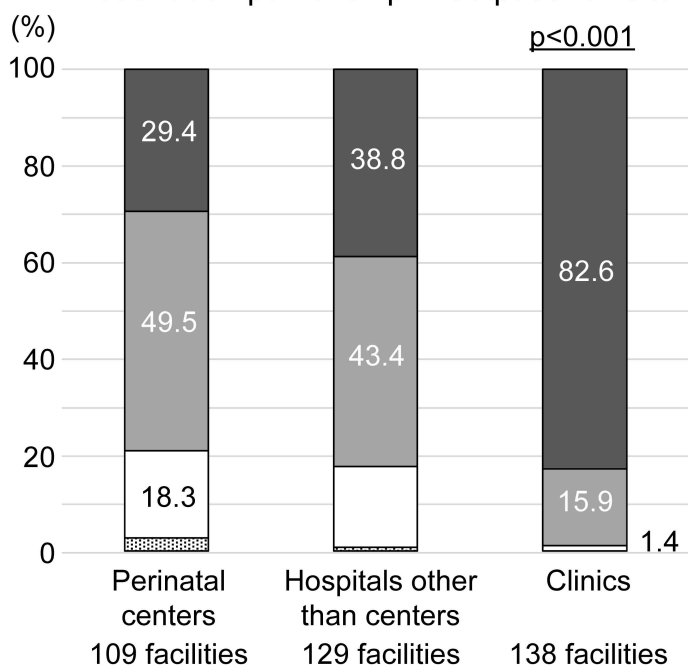

C

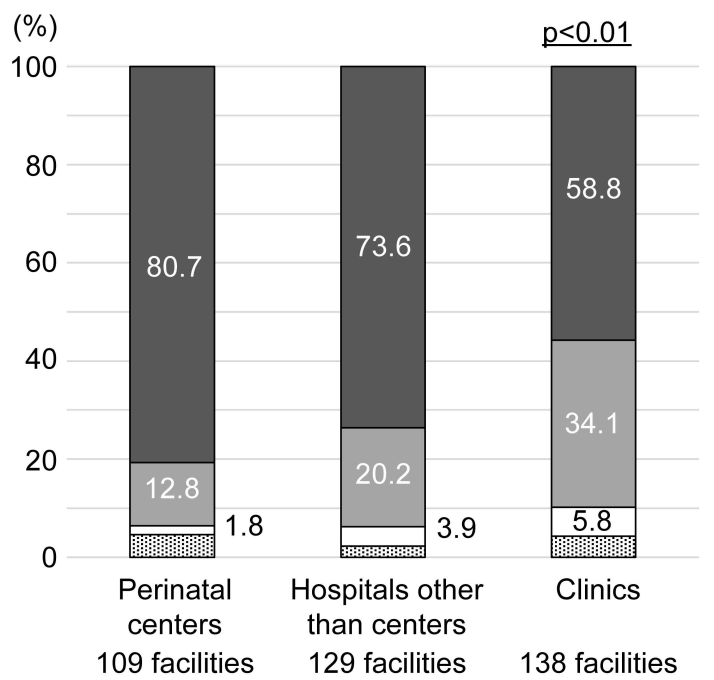

D Patient companionship during delivery


Fig. 2. Changes in the policies and practices of delivery facilities due to COVID-19 pandemic by facility classification. 


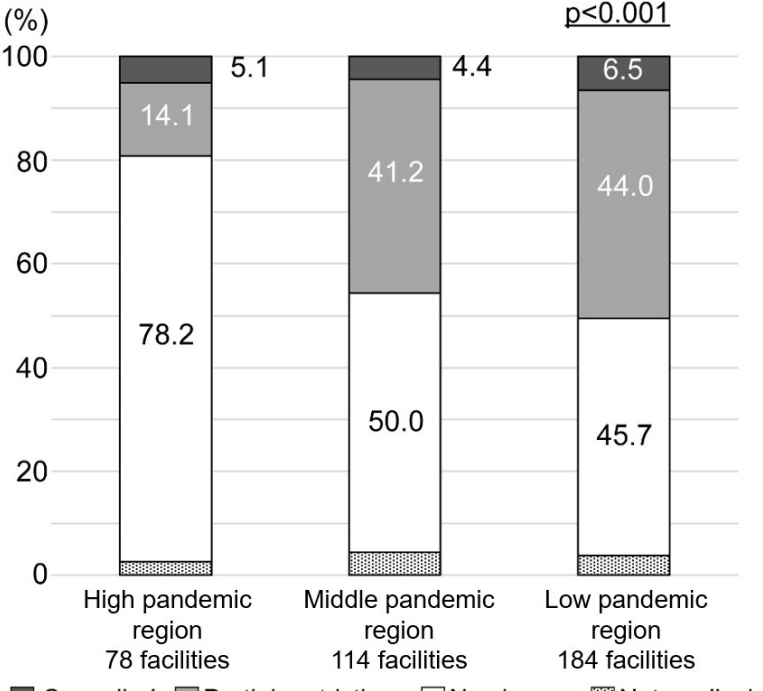

Cancelled $\square$ Partial restrictions $\square$ No change 率 Not applicable

Fig. 3. Status of "satogaeri delivery" acceptance according to the COVID-19 pandemic status by prefectural area classification.

Labour and Welfare in $2017,50.1 \%$ of pregnant women return home for "satogaeri delivery", of which $26.9 \%$ move to a different prefecture [4]. It is estimated that approximately $13 \%$ of pregnant women cross prefectural borders for "satogaeri delivery". It is estimated that a considerable number of pregnant women were affected in 2020. Obata et al. [5] reported that the Edinburg Postnatal Depression Scale (EPDS) scores were higher in pregnant women and postpartum mothers who were unable to go home for delivery. Reasons for not returning home included refusal by the hospital, giving up after talking with the obstetrician, and voluntary giving up owing to infection status [5].

Outpatient visits for pregnant women remained unchanged in about $70 \%$ of the facilities, which had the least impact among the $\mathrm{OB} / \mathrm{GYN}$ practices items surveyed in this study. It was likely considered the minimum necessary care to protect the health of mothers and children. In facilities that had partial restrictions, intervals between medical examinations might have been extended, and medical examination items might have been reduced. In the impact of the COVID-19 pandemic, the implementation of telemedicine and telehealth has become important for the safe and effective healthcare delivery of obstetric care [6]. One study showed that $30 \%$ of pregnant women were as satisfied with or more satisfied with telemedicine than with face-to-face consultations [7]. Approximately $90 \%$ of patient companionships at outpatient visits were canceled or restricted. This would have been detrimental to pregnant women worried about their pregnancy and outpatient visits, and spouses would have lost the opportunity to attend ultrasound exams to share fetal growth.
Patient companionship during labor and delivery was canceled in $67 \%$ of the institutions. The situation was similar for companionship during hospitalization and inpatient visits, meaning that many pregnant women spent time in the hospital from admission for delivery to discharge without family support. Pregnant mothers had to deal with the childbirth period with many physical and mental changes, and spouses were prevented from sharing the experience of the birth of their child. Obata et al. [5] reported that EPDS scores were higher among pregnant women and postpartum mothers from areas with poor infection status. Furthermore, changes in the delivery experience due to the COVID-19 pandemic included not being allowed to have a partner, friend, or other support person attend the birth (33.5\%) and not being able to obtain desired medications before or after the birth (9.7\%). Additionally, inability to receive the desired medications at the time of delivery has been associated with depression [8]. As for the postpartum outpatient clinic, $23 \%$ of the facilities had partial restrictions. This likely makes an already difficult situation harder for expectant mothers who tend to receive poor mental support during pregnancy and are prone to worsening mental health postpartum. A study in the city of Yokohama showed that the overall increase in maternal psychological stress due to the COVID-19 pandemic was not evident. Postpartum women have been reported to react differently in environments with different severity of illness and social constraints [9].

Of the nine items surveyed in this study, no difference was observed by pandemic regions, except for the acceptance of "satogaeri deliveries" mentioned earlier. In other words, it can be said that the same degree of restriction and reduction of maternal care was carried out nationwide, regardless of the status of COVID-19 spread in each area. However, the differences between the facility categories were notable. The percentage of facilities that had canceled either patient companionship during delivery, inpatient visits, or prenatal classes was high among perinatal centers, and decreased in the order of hospitals other than centers and clinics. If a core perinatal facility with a neonatal intensive care unit that admits maternal transport is impaired by a nosocomial infection of COVID-19, perinatal care of the entire region will be affected. Therefore, it is likely that stricter COVID-19 infection control measures were taken at these facilities, which resulted in heavier restrictions on maternal care. It is also possible that general hospitals with other clinical departments that perform surgeries have tighter visitor restrictions. However, nearly half of the clinics continued to allow patient companionship during labor and delivery, albeit with restrictions in place. On the contrary, it is interesting that the rate of cancelation of patient companions for outpatient visits was high in clinics. It is possible that the infection control necessary to have a companion in the outpatient clinic room could not be sufficiently secured, resulting in cancelation. 


\section{A}

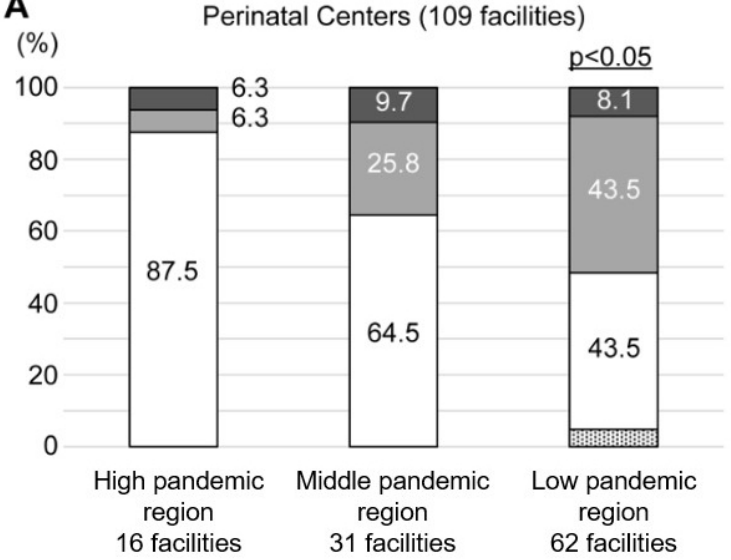

C

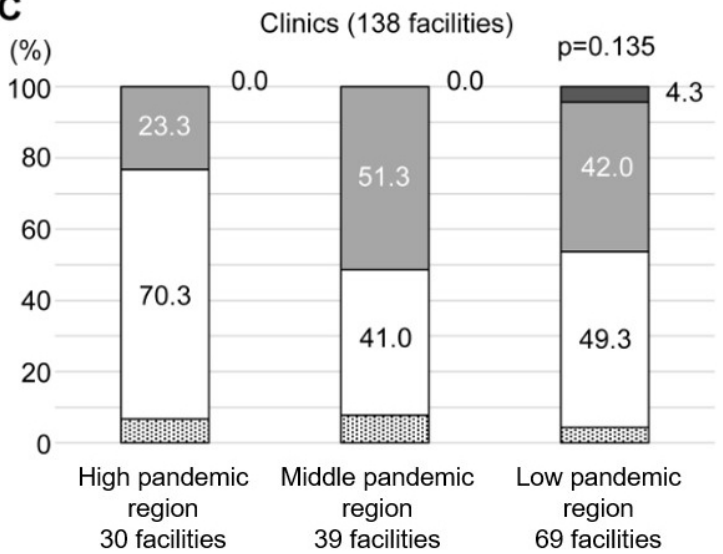

B

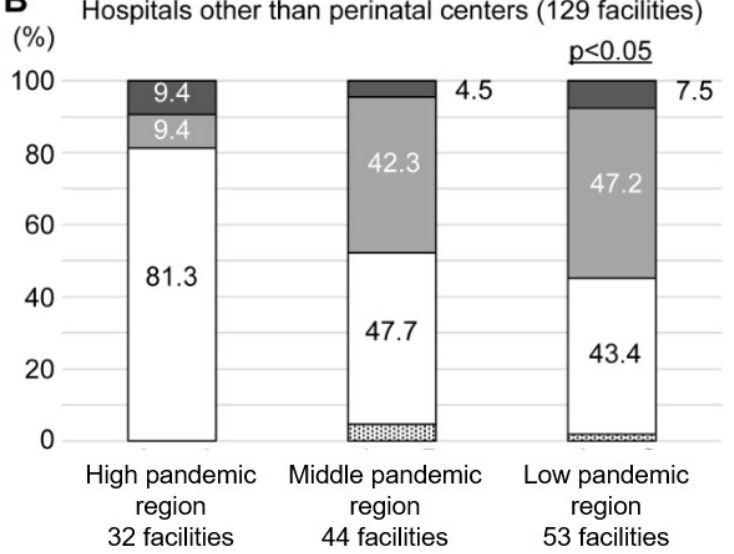

Fig. 4. Status of "satogaeri delivery" acceptance by combined facility classification and the COVID-19 pandemic status.

This study had some limitations. The response rate was low because the survey was conducted at a time when hospitals were extremely busy owing to COVID-19 countermeasures and because there were many items to be answered. Furthermore, our survey was performed at a time when maternal care during the target period was most significantly affected. Therefore, it did not take into account changes over time and periods during which the effect lasted.

\section{Conclusions}

With the COVID-19 pandemic, the differences in the policies and practices of Japan's delivery facilities were more attributed to the role played by facilities in the regional perinatal system than to regional pandemic status. Additional studies are needed to examine the implications of these changes in the policies and practices of delivery facilities on maternal and infant outcomes. This would help reproductive health providers and government policy makers to address the ongoing improvement of reproductive health services under COVID-19.

\section{Author contributions}

Research drafting and planning-YE and MT. Data collection - YE and MT. Data analysis and interpretation-
YE and MT. Manuscript drafts-YE. Critical revision of the manuscript for important intellectual content-YE and MN. Final approval of manuscript - all authors.

\section{Ethics approval and consent to participate}

An online survey was used in this study. Consent for research cooperation was conducted in the form of response submission via Google Forms. This study was carried out with the approval (20-67) of the Institutional Review Board of the Graduate School of Health Sciences, Hokkaido University.

\section{Acknowledgment}

We would like to express our deepest gratitude to all the midwives and ward chiefs nationwide who cooperated in the survey despite being in the busy obstetrics ward during the COVID-19 pandemic.

\section{Funding}

This work was supported by JSPS KAKENHI [grant no: $21 \mathrm{~K} 10818$ ].

\section{Conflict of interest}

The authors declare no conflict of interest. 


\section{Supplementary material}

Supplementary material associated with this article can be found, in the online version, at https://www.imrpre ss.com/journal/CEOG/49/1/10.31083/j.ceog4901006.

\section{References}

[1] Komatsu H, Banno K, Yanaihara N, Kimura T, Board Members of Japan Society of Obstetrics and Gynecology. Prevention and practice during the COVID-19 emergency declaration period in Japanese obstetrical/gynecological facilities. Journal of Obstetrics and Gynaecology Research. 2020; 46: 2237-2241.

[2] Arakaki T, Sekizawa A, Hasegawa J, Ikeda T, Ishiwata I, Kinoshita K, et al. Current status of COVID-19 in pregnant women in Japan: Survey of the Japan Association of Obstetricians and Gynecologist up to June 2020. Acta Obstetrica Et Gynaecologica Japonica. 2021; 73: 597-604. (In Japanese)

[3] Hasegawa J, Arakaki T, Sekizawa A, Ikeda T, Ishiwata I, Kinoshita K. Current status of community-acquired infection of COVID-19 in delivery facilities in Japan. PLoS ONE. 2021; 16 : e0251434.

[4] Ministry of Health. Labour and Welfare, 2017 Child and Childrearing Support Promotion Research Project: Research Report on Health/Medical Cooperation System for Mental Health Care for Pregnant Women. 2018. Available at: https://www.mhlw .go.jp/content/11900000/000520478.pdf (Accessed: 23 August 2021).
[5] Obata S, Miyagi E, Haruyama Y, Umazume T, Kobashi G, Yoshimi A, et al. Psychological stress among pregnant and puerperal women in Japan during the coronavirus disease 2019 pandemic. Journal of Obstetrics and Gynaecology Research. 2021; 47: 2990-3000.

[6] Fryer K, Delgado A, Foti T, Reid CN, Marshall J. Implementation of Obstetric Telehealth during COVID-19 and beyond. Maternal and Child Health Journal. 2020; 24: 1104-1110.

[7] Nakagawa K, Umazume T, Mayama M, Chiba K, Saito Y, Noshiro K, et al. Survey of attitudes of individuals who underwent remote prenatal check-ups and consultations in response to the COVID-19 pandemic. Journal of Obstetrics and Gynaecology Research. 2021; 47: 2380-2386.

[8] Liu CH, Koire A, Erdei C, Mittal L. Unexpected changes in birth experiences during the COVID-19 pandemic: Implications for maternal mental health. Archives of Gynecology and Obstetrics. 2021; 1-11. (in press)

[9] Hiiragi K, Obata S, Misumi T, Miyagi E, Aoki, S. Psychological stress associated with the COVID-19 pandemic in postpartum women in Yokohama, Japan. Journal of Obstetrics and Gynaecology Research. 2021; 47: 2126-2130. 